\title{
Influence of Field Spacer Geometry on the Performance of a High Voltage Coaxial Type Transmission Line with Solid Dielectric Spacer in Vacuum
}

\author{
A. P. Anagha \\ Electrical Engineering Department \\ National Institute of Technology, \\ Calicut, India
}

\author{
K. Sunitha \\ Electrical Engineering Department \\ National Institute of Technology, \\ Calicut, India \\ karakkadsunitha@gmail.com
}

\begin{abstract}
With the development of more powerful high power electromagnetic sources, the transmission of high voltage power particularly between pulsed power systems to huge power rated microwave sources in absence of electrical breakdown and pulse distortion has begun to become a vital issue. A high voltage coaxial type transmission line with $500 \mathrm{kV}$ peak voltage in vacuum is proposed. A novel proposal of configuration bearing a positive angle of $45^{\circ}$ with socket-type filed shaper is being discussed in this paper in a manner to improve surface flashover properties and also to decrease the distortion seen in the pulse shape. This type of transmission line provides high capability of hold off voltage. The electric field distribution of the coaxial geometry with different field shaper configurations and different spacer parameters are also investigated using COMSOL software.
\end{abstract}

Keywords-Field shaper; electromagnetics; surface flashover; triple junction; spacer; dielectrics.

\section{INTRODUCTION}

The development stages of electromagnetic radiation in the wave band regions of microwave and millimeter of electromagnetic spectrum began with the inception of pulsed power technology during the 1960s. The applications of such sources range from plasma physics to communication, some of which being particle acceleration techniques, fusion energy research, high-power radars, etc. [1-5]. The recent trends in the research area of High Power Electromagnetic Sources (HPEM) are basically due to the unmatchable advantages of being capable to deliver high average and peak power [6-8]. Recent trends showcased the development of still more powerful HPEM sources which geared the possibility of transmitting high voltage quasi-square pulses without causing any electrical breakdown. The stress upon electrical breakdown is a quite significant factor in coaxial transmission lines where surface flashover along the dielectric spacer surfaces occurs galore. Therefore, a more detailed study has to be done upon methods to deal with electrical breakdown and pulse distortion phenomenon, for delivering power efficiently along the high power transmission lines using pulsed power devices,.
With proper alteration of the shapes of insulator and with the use of field shapers at triple junctions, significant reduction in surface flashover and dielectric breakdown has been observed. The conventional filed shaper designs are bump type, insertion type and the tangential type [9-13]. The main motive behind the installation of field spacers is to reduce the electric field strength seen at the triple junction where the electrode, dielectric and vacuum all come together. Even though they may seem successful in reducing the huge electric field strength seen at the triple junction, the field strength is not brought down to near zero due to imperfections in the field spacer design. Modification of insulator shape is done by proposing a new design of conical shaped insulator bearing an angle close to $45^{\circ}$ and by increasing the length of surface of the insulator.

Electromagnetic pulse is a short burst of electromagnetic energy that may be originated as a natural or a man-made radiated electric, conducted electric or magnetic field based on the source. The interference of EMP sources is very dangerous to electronic equipment, and high energy EMP sources such as lightning strikes can damage structures like buildings and aircrafts. The medium used to transfer these pulses are mainly grouped as guided and unguided media [4]. Guided media will provide a physical path to propagate the information, which includes twisted pair, coaxial cable and optical fiber. Unguided media uses an antenna to transmit the information through air, vacuum or water. For applications such as high speed local area network and high capacity long distance trunk etc., coaxial cables are used mostly because of their capability of higher data rates over long distances. Hence, the most common type of antenna feeder used today is definitely coaxial cable. The common applications of coaxial cables include: transfer of radio frequency energy, domestic connections between receivers and aerials, carry any level of high frequency signals to any distance etc.

Coaxial cable carries current through both inner and outer conductors, which are equal and opposite and as a result all the fields are confined within the cable. This is the basic principle of cable operation i.e. by propagating an electromagnetic wave 
through it. The nearby objects are not affected by the interferences due to the absence of fields outside the coaxial cable. This is the main advantage of a coaxial cable compared to other forms.

In this paper a new design of coaxial-type transmission line with socket type field shaper and a slight modification of the shape of the dielectric spacer is proposed to minimize the electric field strength and distortion of pulse.

\section{COAXIAL TYPE TRANSMISSION LINE}

The high power coaxial transmission line is composed of both inner and outer conductors, a solid dielectric spacer for supporting the inner structure, and also a field shaper. The conventional and proposed coaxial transmission lines are shown in Figure 1.

\section{A. Basic Design}

Generally, parameters for the coaxial transmission line are based on other factors such as the strength of breakdown field and impedance. Ideally, when losses are neglected, the field distribution pattern inside the coaxial line and also the characteristic impedance are expressed as [3]:

$$
\begin{aligned}
E_{r} & =\frac{v(t)}{r \ln \left(\frac{D}{d}\right)} \\
Z_{\text {coax }} & =\frac{\eta}{2 \pi \sqrt{\varepsilon_{r}}} \ln \left(\frac{D}{d}\right)
\end{aligned}
$$

where $r$ is the radius $(d / 2 \leq r \leq D / 2), v(t)$ is the applied voltage, $d$ and $D$ are the inner and outer diameters of the coaxial line, $\eta$ is the intrinsic impedance, and $\varepsilon_{r}$ is the relative permittivity of the material filling the space between the conductors. As keeping the objective to reduce the electric field strength, it is required to increase the outermost diameter of the coaxial line since the electric field is inversely proportional to the radius $r$. Also, coaxial impedance is proportional to the ratio of the diameter of the inner and outer conductors but inversely proportional to the dielectric permittivity. This makes it necessary to decrease the inner diameter of the coaxial line in order to get an impedance match between the spaces filled with air and dielectric.

\section{B. Surface Flashover Phenomenon}

Insulators ranges in various dimensions are used to supplement the system with potential variations under certain circumstances. This makes the role of High-Voltage hold off capability in insulators to be a vital one vastly in case of practical applications. Vacuum gap will have, mostly, an edge over the solid insulators (of comparable dimension) while considering their High Voltage hold off capacity. The phenomenon of surface discharge can be explained using three different phases: (a) Initiation Phase, (b) Growth Phase, and finally, (c) Final Phase. The instigation of the flashover occurs greatly by the discharge of electrons from the surface of a conductor under the influence of a strong electrostatic field, as a result of tunnel effect, which we call the whole process as Initiation Phase. A very few times, the cause for emission of electrons maybe due to a bunch of electrons or ions hitting the surface of the insulator. There still lies an ambiguity regarding the clarity of explanation for the next phase of event resulting in the growth of discharge, other than a few widely acclaimed interpretations. Most researchers prefer the process of secondary electron emission avalanche mechanism to explain the Development Phase, where a few of the already presen $t$ (emitted) electrons may hit the surfaces and walls of the insulator thereby adding much more electrons into play by secondary emission. This process will continue thus agglomerating the continuously producing electrons to form a series connection of string of electrons called as Secondary Electron Emission Avalanche (SEEA) which is a possible reason for a complete flashover [5].

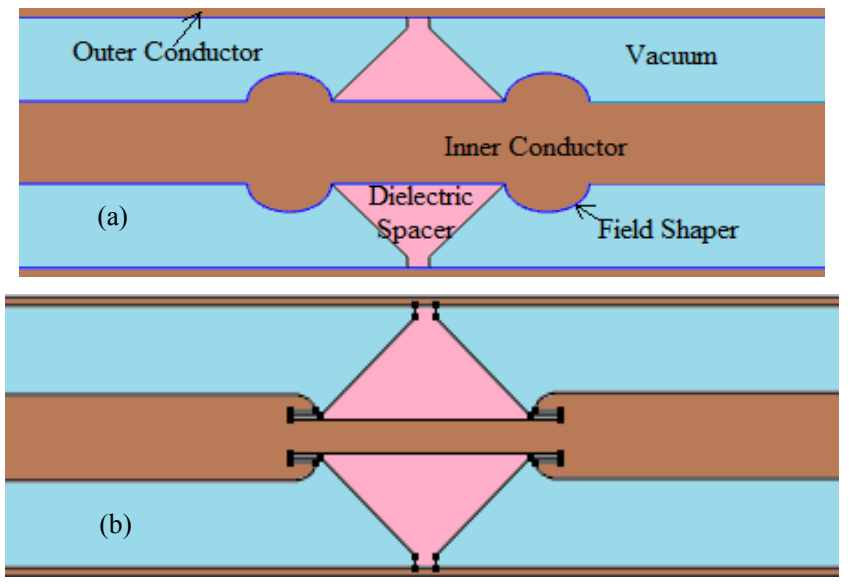

Fig. 1. Coaxial type transmission lines: (a) coaxial geometry with bumptype field shaper, (b) coaxial geometry with socket-type field shaper

The use of High Voltage Vacuum Insulators is mainly in pulsed power generators for the separation of dielectric and vacuum. The vacuum dielectric can be broadly classified as: insulator stack and coaxial insulator; while the commonly used one is the second type mostly in Tesla-type pulse power generator. Our concern in this type of generator is the insulation difficulties which may even lead to the final rupture of vacuum dielectric. The breakdown mechanism can be well reasoned by the aforesaid theory of secondary electron emission avalanche. The breakdown point in the triple junction is shown in Figure 2. The flashover may also be initiated due to anode side electrons at anode triple junction due to which flashover may occur at the surfaces in a predominantly high electric field. This may further increase the electric field intensity on the dielectric surface thus resulting in a huge rupture. This breakdown will build up across the surface on the way towards cathode until the flashover becomes complete [6]. Rather than the dependence alone on the magnitude of the applied voltage, the flashover voltage will greatly depend upon the size and waveform of the voltage. Likely, predominantly short pulses of nanosecond duration will have the highest flashover voltage while the comparatively longer pulses of microsecond duration and also DC pulses will have a medium 
level of magnitude and $\mathrm{AC}$ voltages will be showing the lowest level of magnitude for flashover voltages. The complete time duration of flashover phenomenon depends on the time to initiate while the growth duration will be contributing very less to the total time duration. In addition to the waveform dependence of the flashover voltage, the shape and size of insulator will also exhibit a significant effect on the surface breakdown. Lastly, the type of material also greatly affects the surface flashover phenomenon. Generally, homogenous materials on top of non-homogeneous materials are preferred.

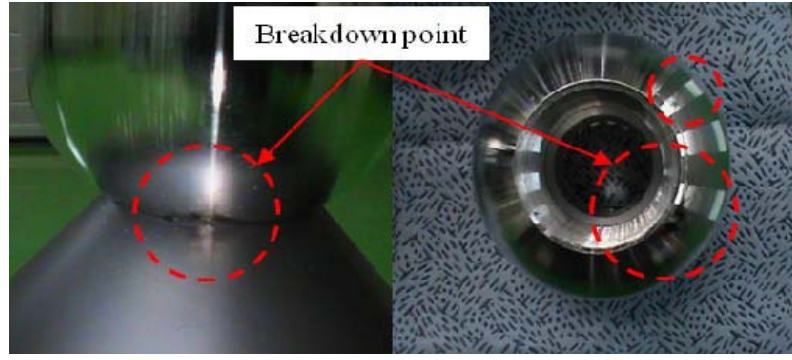

Fig. 2. The breakdown point at the CTJ (Cathode Triple Junction)

\section{DESIGN OF HIGH POWER COAXIAL GEOMETRY}

Different configurations of the coaxial type transmission line are discussed mainly the bump-type and socket type filed shaper design. The materials used for conductor, dielectric spacer and their relative permittivity are shown in Table I. Vacuum is used as the dielectric between the inner and outer conductor. The inner and outer conductor diameters are taken as $102 \mathrm{~mm}$ and $310 \mathrm{~mm}$ respectively [2].

TABLE I. MATERIAL SPECIFICATIONS

\begin{tabular}{|c|c|c|}
\hline Part & Material Used & Relative permittivity \\
\hline Conductor & Stainless steel & 1 \\
\hline Dielectric Spacer & Nylon 66 & 3.2 \\
\hline
\end{tabular}

\section{A. Design of the Bump-type Field Shaper}

The configuration of the bump-type field shaper is shown in Fig. 3. Here, the place for field shaper is around the area of the CTJ where the electrode and dielectric join in the vacuum [7]. This minimizes huge electric field strength experienced around the space. The dielectric spacer is realized using a coaxial type geometry bearing an angle of $45^{\circ}$ to reduce the electric field seen on the dielectric spacer surface.

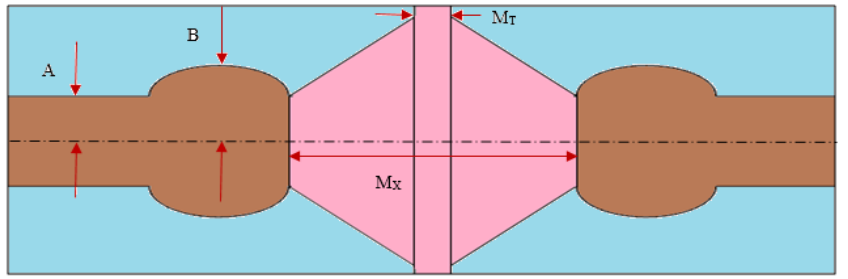

Fig. 3. Configuration of the Bump type field shaper design $\mathrm{M}_{\mathrm{T}} / \mathrm{M}_{\mathrm{X}}=0.096$, $\mathrm{B} / \mathrm{A}=0.843$

\section{B. Design of Socket - type Field Shaper}

Figure 4 shows the improved high power geometry with socket-type field shaper. This is used to make the electric field strength value to almost zero at the CTJ. The main advantage of this field shaper in comparison with the conventional one is that this can conceal the CTJ effectively and also thus it can eliminate the emission seed electron starting at the CTJ. A slight modification of the coaxial type dielectric spacer bearing a positive angle of $45^{\circ}$ is done at both ends to enhance the support provided to both inner and outer conductors. This modification also makes it advantageous by effectively shielding the cathode structure and therefore keeps away the dielectric surface from the huge electric field around the region of the cathode field shaper.

(a)

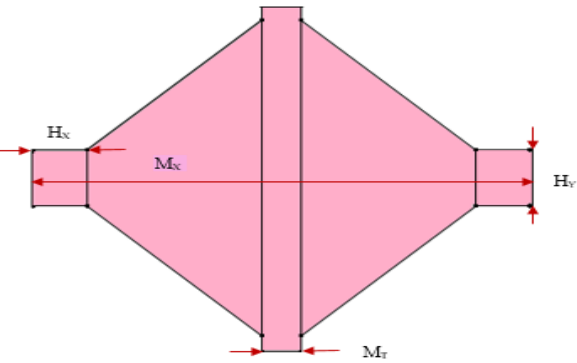

(b)

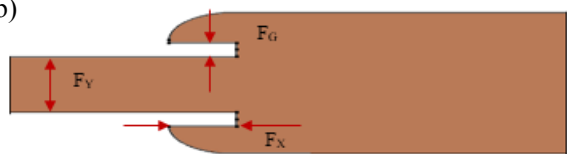

Fig. 4. Coaxial solid dielectric spacer $\mathrm{M}_{\mathrm{T}} / \mathrm{M}_{\mathrm{X}}=0.079, \mathrm{H}_{\mathrm{Y}} / \mathrm{H}_{\mathrm{X}}=1.9$ and (b)socket type field shaper with $\mathrm{F}_{\mathrm{x}} / \mathrm{F}_{\mathrm{y}}=0.375, \mathrm{~F}_{\mathrm{G}} / \mathrm{F}_{\mathrm{Y}}=0.25$

\section{Modified Design of Socket type Field Shaper}

A modified design of the socket type field shaper is proposed in this paper to reduce the electric field mainly at the portion where the dielectric spacer and outer conductor meet. A socket type field shaper is used at CTJ. Instead of conical shaped dielectric with a positive angle of $45^{\circ}$ a curved shape spacer is used. The proposed design is shown in the Figure 5.

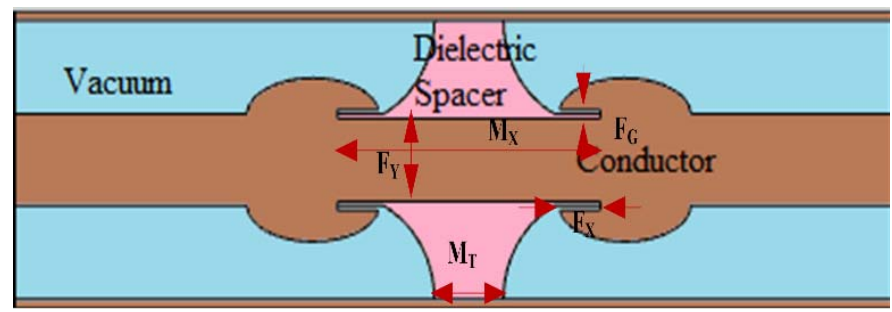

Fig. 5. Modified design of socket type field shaper: $F_{x} / F_{y}=0.375$, $\mathrm{F}_{\mathrm{G}} / \mathrm{F}_{\mathrm{Y}}=0.25, \mathrm{M}_{\mathrm{T}} / \mathrm{M}_{\mathrm{X}}=0.179$ and curved shape spacer

\section{ANALYSIS OF HIGH POWER COAXIAL GEOMETRY}

To compare and verify the conventional and proposed high power coaxial geometry, simulation of different designs are carried out using the COMSOL MULTIPHYSICS software. 
Electrostatic analysis of the different configuration is performed. An excitation of $500 \mathrm{kV}$ is applied to the inner conductor and the outer conductor is grounded.

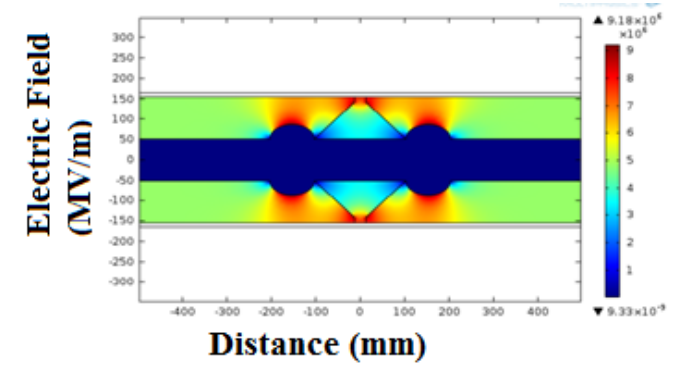

Fig. 6. Electric field distribution of Bump type field shaper

The electric field distribution of the bump type and of the socket type design is shown in Figures 6 and 7 respectively. The electric field distribution of socket type field shaper is more uniform compared to bump type field shaper along the coaxial solid dielectric. The bulged shape of the bump type field shaper makes the distribution more non-uniform and larger electrical stress is experienced in these regions.

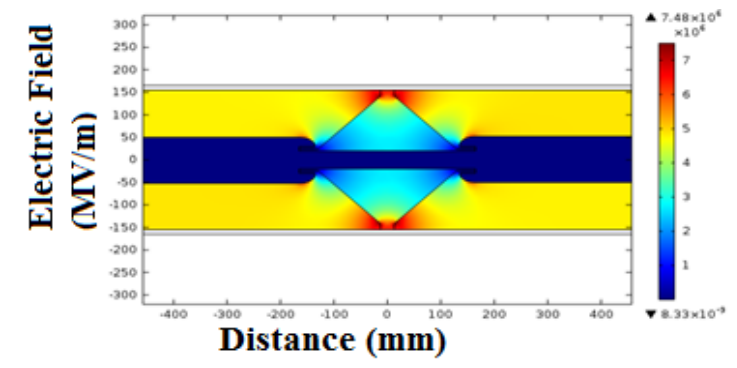

Fig. 7. Electric field distribution of Socket type field shaper

\section{A. Analysis of Bump type Design}

The dimensions of the bump shape are varied and the electric field distribution is observed. It is analyzed that as the bulged shape of the bump decreases, the electric field strength on its surface decreases and it becomes more uniform also. The simulation results are given in Figure 8. We can clearly observe from the results that, the intensity of the shape of bumpiness and the electric field strength are inversely proportional.

\section{B. Analysis of Socket type Design}

The bulged shape is completely removed and a new type of design called socket type design is proposed.

\section{1) Effect of Spacing Width of Socket}

The spacing width of the socket is varied and the electric field distribution is simulated which is shown in Figure 9. It is observed that a large variation in field distribution is not observed while varying the spacing, but a slight increase in the field strength as spacing decreases is analyzed. Spacing dimensions affect least importantly in the case of analysis of the electric field strength and they pose minimum threat to the design scenario with regard to electric field strength. But always a minimum spacing has always to be maintained for safer field strength levels.

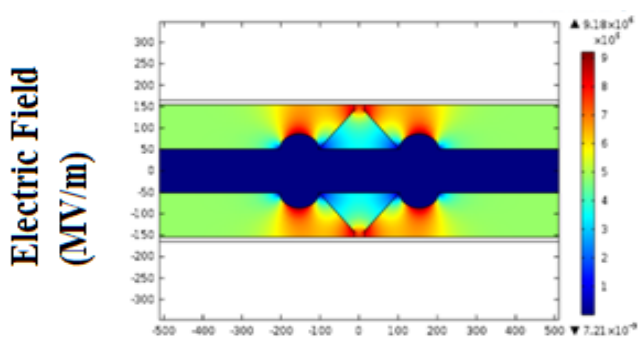

Distance (mm)
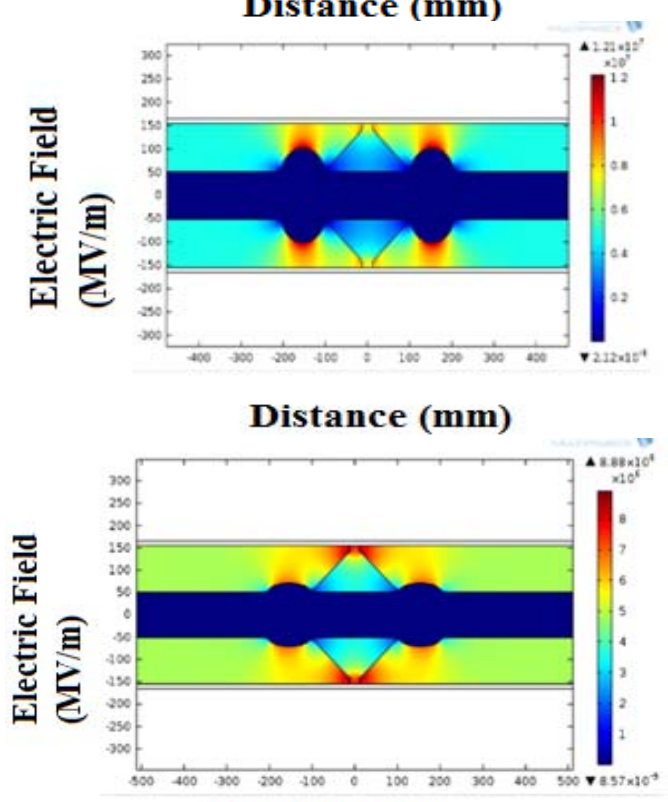

Distance (mm)

Fig. 8. Analysis of bump type design

\section{2) Effect of radius of the socket}

The radius of the socket is also varied and observations are shown in Figure 10.As the radius of the socket decreases a large increase in the electric field strength is observed. Electric field strength and the socket radius length are again inversely proportional and therefore the radius has to be maintained at a safe level so as to reduce the effect of high electric field strength at that region.

\section{Analysis of Proposed Modified Design}

From comparison, it is observed that electric field distribution on the surface of the cathode filed shaper is reduced to a large extend in case of socket type field shaper design. But both geometries have high electric field strength at the portion of dielectric spacer which meets the outer conductor. In order to overcome this drawback a modified spacer design of socket type field shaper coaxial geometry is proposed. The electric field distribution of the proposed design is given in Figure 11. A moreover uniform electric field 
distribution is achieved by using the modified design of coaxial geometry. The simulation results for various dimensions of the new design are given in Figure 12. From the results it is clear that spacer with wider gap has uniform field distribution. The other one has large field concentration at the portion where spacer and outer conductor meet.

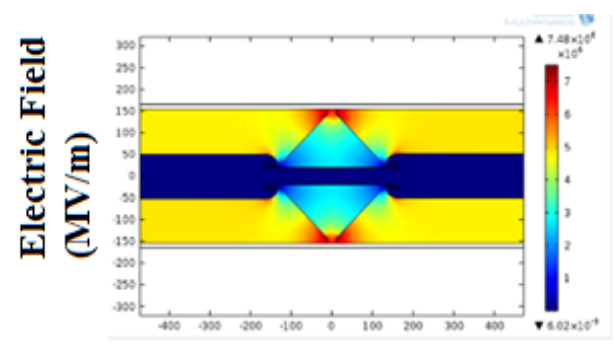

Distance (mm)

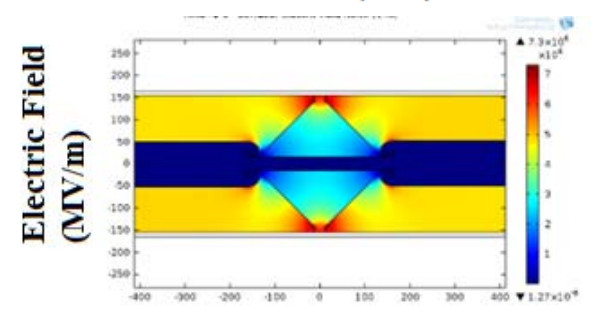

Distance (mm)

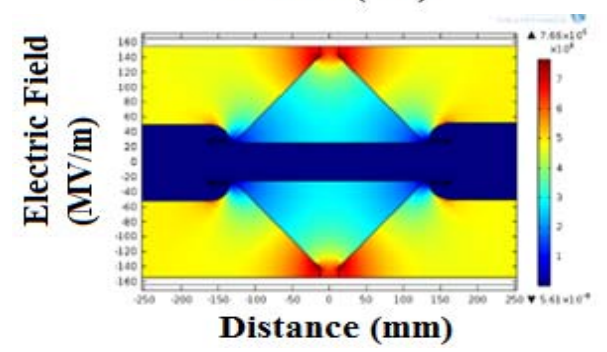

Fig. 9. Variation of Electric field with spacing of the socket
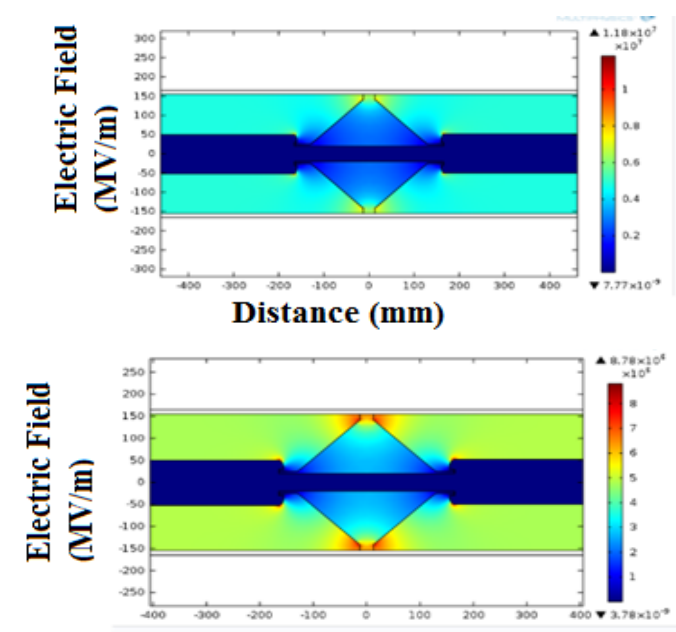

Distance (mm)

Fig. 10. Variation of electric field with radius of the socket

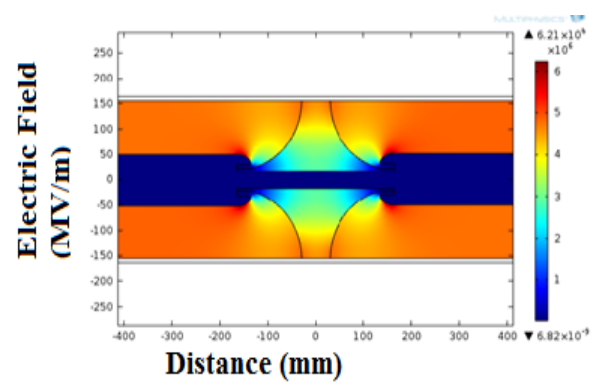

Fig. 11. Electric field distribution of the modified design
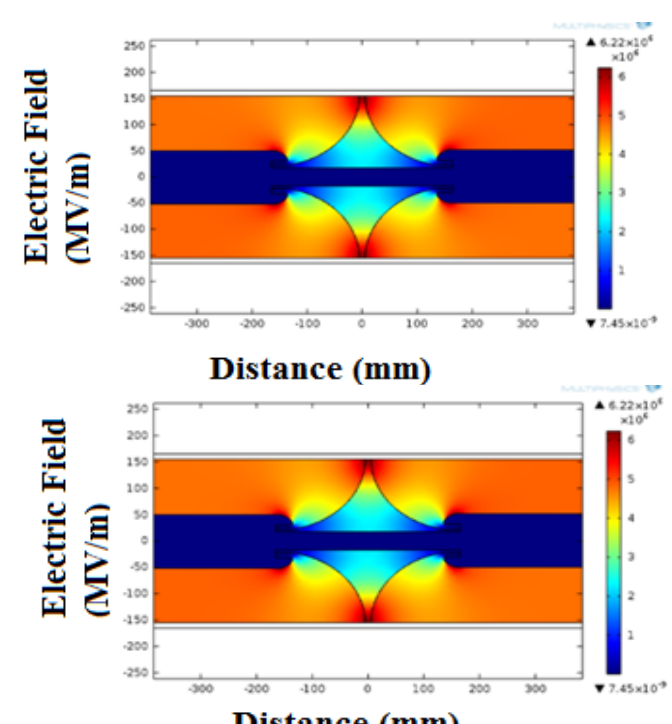

Distance (mm)

Fig. 12. Electric field for the modified design of spacer

\section{Comparison of all designs}

Comparing the electric field distribution of bump, socket and modified designs, it is observed that field distribution is more uniform with socket type field shaper and circular shape dielectric spacer. The range of maximum electric field as well as the minimum electric field is least for proposed modified design. The electric field is highest for bump type geometry and least for the modified geometry. This fact is better illustrated in Table II.

TABLE II. RESULTS COMPARISON

\begin{tabular}{|c|c|c|}
\hline Field shaper geometry & Dielectric spacer & $\begin{array}{c}\text { Difference between } \\
\text { maximum and } \\
\text { minimum field } \\
\text { (MV/m) }\end{array}$ \\
\hline Bump type & Rhombus type & 91.7 \\
\hline Socket type & Rhombus type & 74 \\
\hline Proposed modified design & Circular curved & 6 \\
\hline
\end{tabular}

\section{CONCLUSION}

A coaxial-type matched transmission line to transmit high power with reduced electrical breakdown and pulse distortion is proposed and analyzed. A comparison of different field 
shaper designs is carried out and concluds that the socket type field shaper is more favorable. Finally a modification is done for the spacer shape in the socket type filed shaper design. This has the basic advantage of reducing the electric field at the position where both spacer and outer conductor meet. This type of transmission line that can provide a significant reduction of electric field strength to near zero at the CTJ and also good pulse shape may be very useful for high voltage pulse power applications.

\section{REFERENCES}

[1] S. D. Korovin, V. V. Rostov, S. D. Polevin, I. V. Pegel, E. Schamiloglu, M. I. Fuks, R. J. Barker, "Pulsed power-driven high-power microwave sources", Proceedings of the IEEE, Vol. 92, No. 7, pp. 1082-1095, 2004

[2] R. Kichouliya, M. Joy Thomas, "Interaction of high power electromagnetic pulses with power cables and electronic systems", IEEE International Symposium on Electromagnetic Compatibility, Canada, July 25-29, 2016

[3] J. Choi, Y. Kim, J. So, "Design of low distortion high voltage coaxialtype transmission line for quasi-square wave nano-pulse with several hundred kilovolts in vacuum", IEEE Transactions on Dielectrics and Electrical Insulation, Vol. 22, No.4, pp. 1809 - 1814, 2015

[4] S. F. Mahmoud, Electromagnetic waveguides: theory and applications, IET, 1991

[5] H. C. Miller, "Surface flashover of insulators", IEEE Trans. Electr. Insul., Vol. 24, No. 5, pp. 765-786, 1989

[6] L. Zhao, J.-C. Peng, Y.-F. Pan, X.-B. Zhang, J.-C. Su, "Insulation analysis of a coaxial high-voltage vacuum insulator," IEEE Trans. Plasma Sci., Vol. 38, No. 6, pp. 1369-1374, 2010

[7] J. Choi, Y. Kim, J. So, "Design and experiments of the $600 \mathrm{kV}$ coaxialtype matched divider for high-power microwave source array", IEEE Trans. Electron Devices, Vol. 61, No. 12, pp. 4240-4246, 2014

[8] L. G. Christophorou, R. V. Van Brunt, J. K. Olthoff, "Sulfur Hexafluoride and the electric power industry", IEEE Electrical Insulation Magazine, Vol. 13, No. 5, pp. 20-24, 1997

[9] L. Niemeyer, F. Y.Chu, "SF6 and the atmosphere", IEEE Transactions on Electrical Insulation, Vol. 27, No. 1, pp. 184-187, 1992

[10] L. G. Christophorou, R. V. Van Brunt, "SF6 /N2 mixture basic and HV insulation properties", IEEE Transactions on Dielectrics and Electrical Insulation, Vol. 2, No. 5, pp. 952-1003, 1995

[11] T. Nitta, Y. Shibuya, Y. Fujiwara, Y. Arahant, H. Takahashi, H. Kuwahara, "Factors controlling the surface flashover in SF6 gas insulated systems", IEEE Transactions on Power Apparatus and Systems, Vol. PAS-97, No. 3, pp. 959-965, 1978

[12] T. S. Sudershan, R. A. Dougal, "A review of mechanism of surface flashover along solid dielectrics in compressed gases", IEEE Transactions on Electrical Insulation, Vol. EI-21, No. 5, pp. 727-746, 1986

[13] J. A. Bakken, "Determination of characteristics of voltage in impulse and switching surge testing", IEEE Transactions on Power Apparatus and Systems, Vol. PAS-86, No. 8, pp. 962-968, 1967 